\title{
Postacute management of patients with spinal cord injury due to metastatic tumour disease: survival and efficacy of rehabilitation
}

\author{
D Parsch ${ }^{1}, \mathrm{R} \mathrm{Mikut}^{2}$ and R Abel*,1 \\ ${ }^{1}$ Orthopaedic University Hospital Heidelberg, Heidelberg, Germany; ${ }^{2}$ Institute for Applied Computer Science, \\ Karlsruhe, Germany
}

\begin{abstract}
Study design: Retrospective study utilising clinical records and public administration databases.

Objectives: This study was performed to analyse the clinical presentation and survival rate of individuals with spinal cord injury (SCI) due to spinal metastasis after primary treatment, and to evaluate the efficacy of rehabilitative efforts.

Setting: Spinal Cord Injury Unit, University Hospital, Heidelberg.

Methods: A total of 68 consecutive patients were included. Demographics, clinical data, tumour type, level and completeness of SCI, initial treatment, functional independence measure (FIM) and survival time were derived from hospital and public administration databases. Cox regression and fuzzy logic rule generation were used for statistical analysis.

Results: Of the 68 patients, 66 patients died 11 months (median, interquartile range (IQR) 4-29 months) after the onset of neurological symptoms at an average age of 58 years. The functional independence measure (FIM) score describing the general clinical and functional status proved to be the most reliable prognostic factor of survival. Other more specific parameters (eg tumour type or level of lesion) did not have such an impact. In total, 51 patients completed the rehabilitation programme within 50 days (median, IQR 27-99 days). The FIM score improved from 62 at admission to 84 at discharge.

Conclusion: The clinical and functional status is a valuable prognostic factor for survival. Since institutionalised rehabilitative efforts are effective, this group of patients should be accepted into such a program.
\end{abstract}

Spinal Cord (2003) 41, 205-210. doi:10.1038/sj.sc.3101426

Keywords: spinal cord injury; spinal metastasis; survival; rehabilitation

\section{Introduction}

Spinal cord injury (SCI) is a disabling complication of cancer; symptomatic spinal metastases are reported in up to $5 \%$ of all cancer patients. ${ }^{1}$ Clinical symptoms of SCI include severe back pain, sensory deficits, autonomic sphincter control impairment, and weakness or paralysis of the extremities. The onset of neurological deficits is considered to be a medical emergency. Various treatment protocols are reported, including surgical treatment, ${ }^{2-8}$ radiation therapy ${ }^{2,9,10}$ and chemotherapy. ${ }^{11,12}$ An improvement of neurological deficits has been documented in up to $80 \%$ of cases. This 'success rate' implicates a group of patients with persisting or deteriorating neurological symptoms. The physician in charge has to decide whether these patients qualify for rehabilitative efforts. An individual

*Correspondence: R Abel, Orthopaedic University Hospital Heidelberg, Schlierbacher Landstrasse 200; D-69118 Heidelberg, Germany assessment is mandatory to prevent unnecessary hospitalisation of terminally ill patients and to provide an adequate rehabilitation programme for patients with good prognosis. Essential questions are: How long will they survive? Are there prognostic factors? What is, after all, the efficacy of a rehabilitation programme for these patients?

The aim of the present study is to (1) evaluate the survival time of patients with SCI due to spinal metastasis after primary treatment, (2) identify prognostic factors for longer-term survival, and (3) analyse the efficacy of a rehabilitation programme for this special group of patients.

\section{Methods}

Using the medical computerised database of our SCI unit, 68 (30 males and 38 females) consecutive inpatients 
with SCI due to tumour metastases of the spine were identified. To assure a minimum follow-up of 5 years, the search was limited to the time interval between 1979 (institution of the database) and 1995. All patients had received primary treatment appropriate to the clinical situation. Spinal surgery, radiation therapy, chemotherapy or combined treatment (Table 1) was performed, but still substantial neurologic deficits were present. Patients with single nerve root or plexus lesions were excluded.

Public administration databases were used to retrieve the current address of survivors or the date of death. The data extracted from the medical charts included date of birth, gender, type of tumour and primary treatment, dates of tumour diagnosis and onset of neurologic symptoms, a description of the neurologic deficit (level of lesion, Frankel classification), as well as the dates of admission and discharge.

To assess the functional status of our patients, the detailed reports of the occupational and physical therapists were used to retrospectively determine the functional independence measure (FIM) ${ }^{13}$ score at admission as well as at discharge for 51 patients who completed the rehabilitation programme. Additionally the patient's mobility was categorised into 'bedridden', 'wheelchair user' and 'minimum walking ability preserved'. A total of 17 patients did not complete the rehabilitation programme: in 11 cases medical problems necessitated a transfer to another hospital, and six patients died in the ward.

Finally, the presence of pressure sores at the time of admission and discharge was noted. The treatment offered in our full-scale SCI unit (total capacity of 60 beds) was focused on effective pain control, voiding and bowel management, treatment of pressure sores, mobilisation by physical therapists, and the adaptation of aids (eg wheelchair, mattress) by occupational therapists. The social service was involved in the postrehabilitation placement of the patients. Whenever necessary, psychological counselling was offered. Our rehabilitation efforts were intended to be completed within 4-6 weeks. Survivorship was estimated using the Kaplan-Meier methods. The following parameters were included for statistical analysis:

Table 1 Treatment of spinal lesion

Treatment $N$

Radiation therapy only

Chemotherapy only

Surgery only

Radiation therapy and chemotherapy

Radiation therapy and surgery

Chemotherapy and surgery

Radiation therapy, chemotherapy and surgery

No specific treatment for the spinal lesion

Total

\section{2}

2

10

11

12

4

13

4

68
Gender (male-female)

Mobility (bedridden-mobile (wheelchair, walking ability))

Frankel scale (A-B-C-D)

Type of tumour (risk groups I, II and III)

FIM at admission (quantitative)

Level of SCI (quantitative)

Age at the time of SCI (quantitative)

Cox regression and fuzzy logic rule generation were used to identify prognostic factors. Cox regression analysis was performed using Statistica for Windows (Statsoft, Tulsa, OK, USA). Stepwise procedures were used, either to select (forward selection) or to exclude parameters (backward selection) from a given set of variables (see above). A significance level of $P<0.05$ was used.

Fuzzy logic was introduced by $Z_{\text {adeh }}{ }^{14}$ and is now an established method to process uncertain information. The main purpose of a fuzzy system consists in modelling and explanation of the general dependencies between the system inputs and outputs. Fuzzy systems can be built by transforming expert knowledge into linguistic terms and rules. Recently, research was directed towards automatic data-based design methods for fuzzy classifiers, ${ }^{15,16}$ which were also used in this project.

Fuzzy classifiers consist of three components:

- fuzzification (conversion of measurement data $x$ into a linguistic description of this value),

- inference (processing the linguistic result of the fuzzification and linguistic IF-THEN rules to a linguistic result) and

- defuzzification (conversion of this linguistic result into a quantitative value).

The advantages of fuzzy classifiers in comparison to multivariate techniques are:

- the generation of well-interpretable IF-THEN rules,

- the possibility of finding nonlinear and multivariate dependencies,

- the easy handling of mixed quantitative (eg FIM) and qualitative (eg tumour types) features and

- a quantitative estimation of output values (eg for individual survival times).

\section{Results}

\section{Survival}

The search in public administration databases allowed follow-up for all patients. Two male patients were alive at follow-up; 66 patients had died with an average age of 57.5 years (SD 15.5; range $6.2-83.5$ years). The average age at the time of tumour diagnosis was 52.3 years (SD 15.6; range 2.5-82.9 years), and at the time of SCI 54.9 years (SD 15.6; range 2.5-82.9 years).

When presenting with neurologic deficits, the tumour diagnosis had been established for 3.5 months (median; inter quartile range (IQR) 0-41 months); for 32 patients SCI was the primary manifestation of the tumour.

The median survival was 11 months (25th percentile 4 months, 75th percentile 27 months; Figure 1) after SCI 


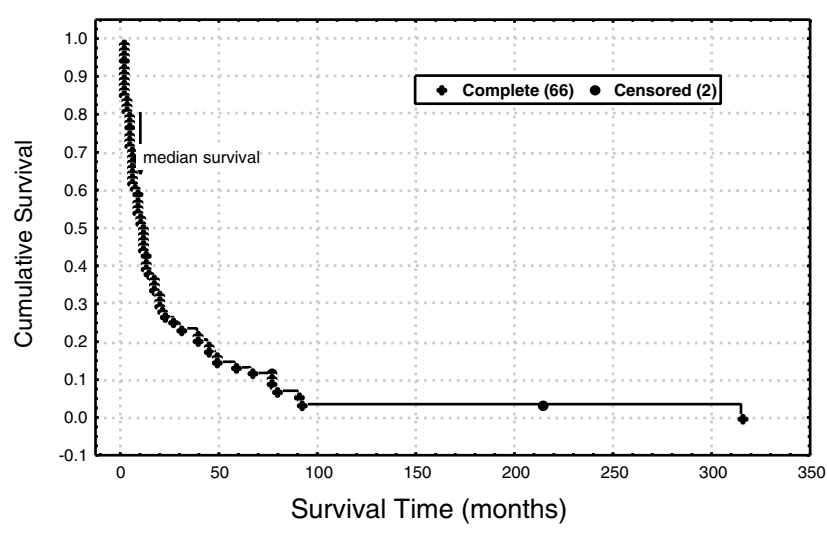

Figure 1 Survival function following SCI $(n=68)$

Table 2 Tumour type (according to pathology report) and frequency

Type of tumour

$n$

Thyroid neoplasm

Malignant teratoma

Collum neoplasm

Non-Hodgkin lymphoma

Nephroblastoma

Breast carcinoma

Chondrosarcoma

Hodgkin lymphoma

Prostatic carcinoma

Plasmocytoma

Esophagea neoplasm

Adrenal gland neoplasm

Kidney neoplasm

Liposarcoma

Gallbladder carcinoma

Bronchial neoplasm

Colon carcinoma

Ewing sarcoma

Unknown

and 38 months (25th percentile 9 months, 75 th percentile 73 months) after tumour diagnosis. According to the purpose of this study, we use the survival after SCI for the subsequent data analysis.

\section{Tumour type}

The most frequent origins of tumour were breast, lung, kidney and prostate. Patients with multiple myeloma were included. In three cases, the primary tumour site was unknown. All tumours were classified according to reported 5-year survival rates of more than 50\% (risk group I, eg thyroid carcinoma, lymphoma), 20-50\% (risk group II, eg breast carcinoma) and less than $20 \%$ (risk group III; eg lung carcinoma, gastrointestinal carcinoma, unknown). ${ }^{7,17-19}$ Table 2 summarises the data on the spectrum of tumour pathology.

In 18 cases metastasis to sites other than the spine was known. However, since the diagnostic procedures employed to determine the presence or absence of further tumour varied widely, this information cannot be used for further statistical analysis.

\section{Level and severity of SCI}

The cervical spine was involved in seven cases. In sum, 59 thoracic and two lumbar lesions were seen (Figure 2). Complete loss of sensory and motor function (Frankel A) was found in 21 patients, and incomplete sensory loss and complete loss of motor function (Frankel B) in 11 patients. Some motor function (without functional use) and incomplete sensory loss (Frankel C) was diagnosed in 24 patients, and useful motor function with incomplete loss of sensory function (Frankel D) in 12 patients.

\section{Mobility and FIM}

A total of 55 patients were bedridden, and 13 were mobile (10 using a wheelchair and three with walking ability). The FIM score at admission was 62 (IQR 56-70). Completion of the rehabilitation programme was possible for 51 patients, and the characteristics of the 17 cases in which the rehabilitation could not be completed are discussed below.

The data used in the following section were derived from the group of patients completing the rehabilitation programme. Their median length of hospital stay was 50 days (IQR 27-99 days). For these patients, the FIM score improved from 62 at admission (IQR 58-72) to 84 at discharge (IQR 66-105), resulting in a rehabilitation efficiency $^{20}$ (FIM difference/length of stay) of 0.33 (IQR 0.06-0.60). The improvements in the subscores of the FIM are given in detail in Figure 3.

The median length of hospital stay was also investigated for patients completing the rehabilitation. It was 42 (IQR 28-87) days for 43 patients without pressure sores. It increased significantly for the eight patients who presented with pressure sores to 123 (IQR 56-156) days ( $t$-test, $P<0.05$ ). The FIM score of patients with pressure sores at admission was 60 (IQR 57-71), and at discharge 87 (IQR 62-108). The rehabilitation efficiency for these patients was 0.23 (IQR $0.08-0.36$ ).

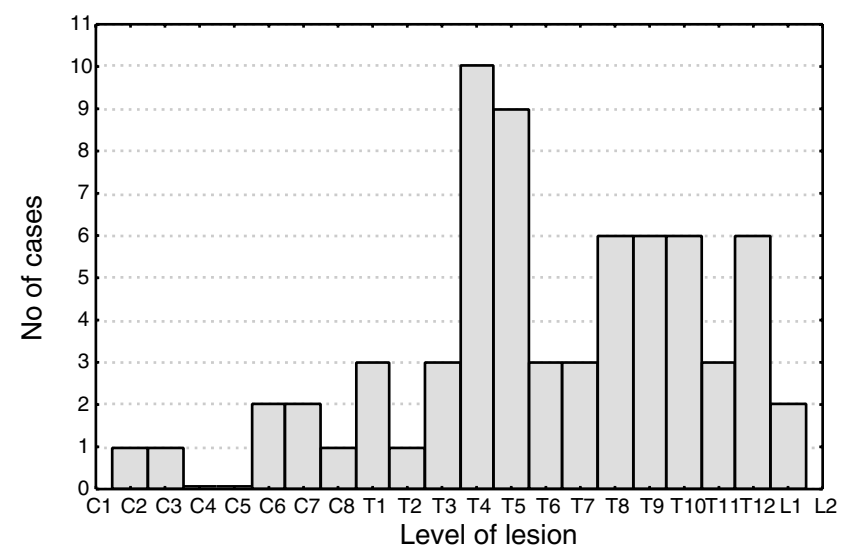

Figure 2 Level of SCI $(n=68)$ 
Patients not completing the rehabilitation programme

A total of 17 patients did not complete the rehabilitation programme: six patients died in the ward and 11 were transferred to another hospital for varying medical reasons. Six patients died on the ward at a mean age of 44.1 years. Their initial FIM score was 57 (median). Three of these patients presented with pressure sores.

The patients transferred were not different from the population completing the rehabilitation in any of the parameters given above. The reasons for transfer included unexpected secondary tumour treatment (radiation, chemotherapy); two patients requested transfer to a hospital closer to their home.

\section{Statistical analysis}

Multivariate analysis using Cox regression Forward selection revealed that a higher FIM score at admission, female gender, lower age at the time of SCI and 'incomplete neurological deficit' (Frankel B, C and D) are positive prognostic factors. Backward selection confirms these results. Detailed results are given in Table 3, which gives the $P$-values and the relative risks for all factors in the resulting model. Using Cox regression analysis, the FIM proved to be the most reliable prognostic factor: a higher FIM score is associated with longer survival. In addition, incomplete SCI (Frankel B,

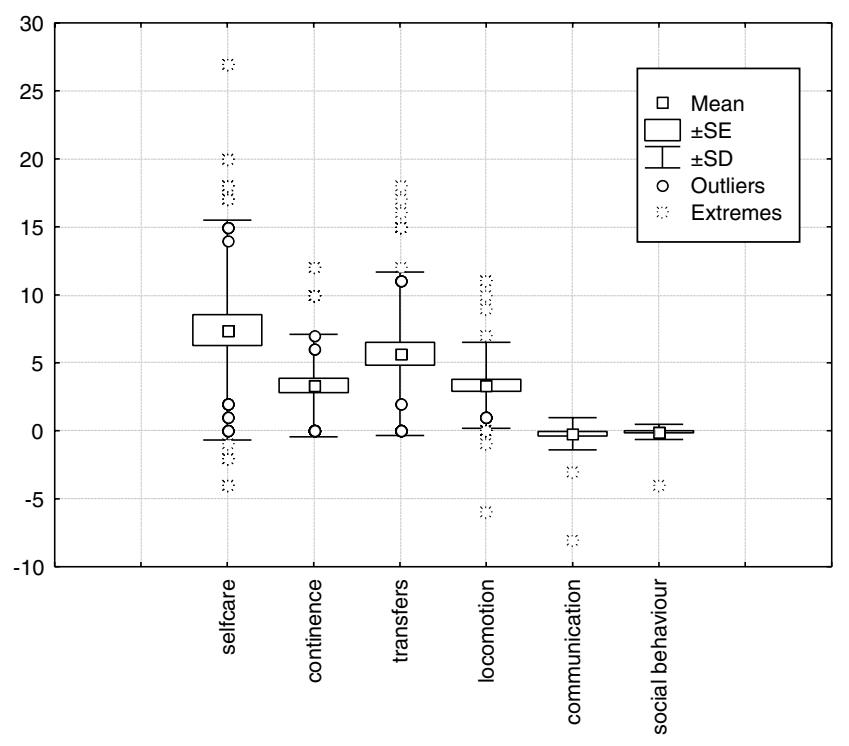

Figure 3 Functional improvement in FIM subscores
$\mathrm{C}$ and $\mathrm{D})$ gives a better prognosis compared to complete SCI (Frankel A). Younger age at the time of SCI and female gender also increase the chance for longer survival.

Fuzzy classification The most important and subsequently selected fuzzy rules to discriminate between longer ( $>10$ months; $n=35$ ) and shorter ( $<10$ months; $n=33$ ) survival were the following:

- A good or very good FIM score at admission (approximately $>65$ ) indicates longer survival (Figure 4).

- A poor or very poor FIM score at admission $(\leqslant 65)$ indicates short-term survival (Figure 4).

- Low Frankel classification (B, C, D) and not very aggressive tumours are indicators of longer survival (Figure 5).

- Wheelchair mobility or walking ability also indicate longer-term survival.

The fuzzy classifier works with an error level of $17 \%$ in the detection of the two output classes long-term and short-term survival using training data and $22 \%$ processing test data compared to the chance selection error of approximately $50 \%$. If we follow a varification strategy via cross validation and binomial distributions, the quality of the whole fuzzy classifier can be proven with a $P$ value of $1 \times 10^{-6}$.

\section{Discussion}

Traditionally SCI units were established to treat and rehabilitate trauma patients. The efficacies of these treatment protocols are widely accepted and well documented. ${ }^{21-24}$ Despite the large number of patients with spinal metastasis ${ }^{19,25}$ there has always been a hesitancy to refer and admit these patients to dedicated SCI units for rehabilitation. Reasons are the general opinion that extensive rehabilitative efforts may either not be tolerated by the terminally ill patient or be futile because of the limited life span. While there are several studies that report the outcome of primary treatment and recommend an aggressive approach, ${ }^{3-8}$ there is only limited information about postacute management. ${ }^{20}$ In 1996 McKinley et $a l^{26}$ documented the efficiency of rehabilitation in a larger series (32 patients). Offering an interdisciplinary team approach, the authors reported a significant functional improvement documented by increasing FIM values. The results of our series of 68

Table 3 Results derived from Cox regression analysis

\begin{tabular}{|c|c|c|c|c|c|}
\hline & $\beta$ & Standard & t-value & Exponent & $P$ \\
\hline Gender & -0.609746 & 0.267366 & -2.28056 & 0.543489 & 0.022581 \\
\hline Age & 0.016138 & 0.008081 & 1.99708 & 1.016268 & 0.045825 \\
\hline FIM at admission & -0.026796 & 0.009627 & -2.78343 & 0.973560 & 0.005382 \\
\hline Frankel & 0.979664 & 0.302691 & 3.23652 & 2.663562 & 0.001211 \\
\hline
\end{tabular}

$\chi^{2}=25.0174, \mathrm{df}=4, P($ model $)=0.00005$ 


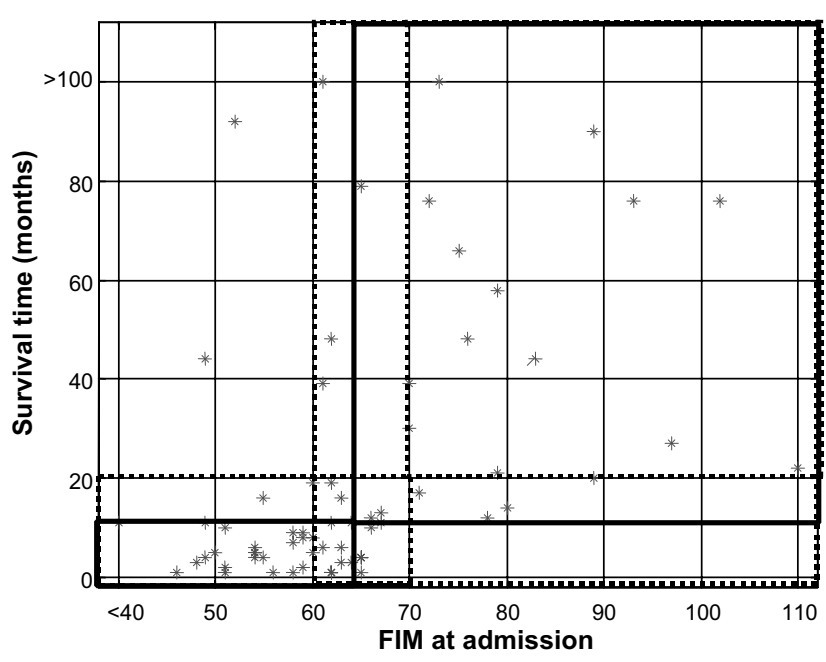

Figure 4 Survival time dependent on FIM (bold lines: fuzzy rules)

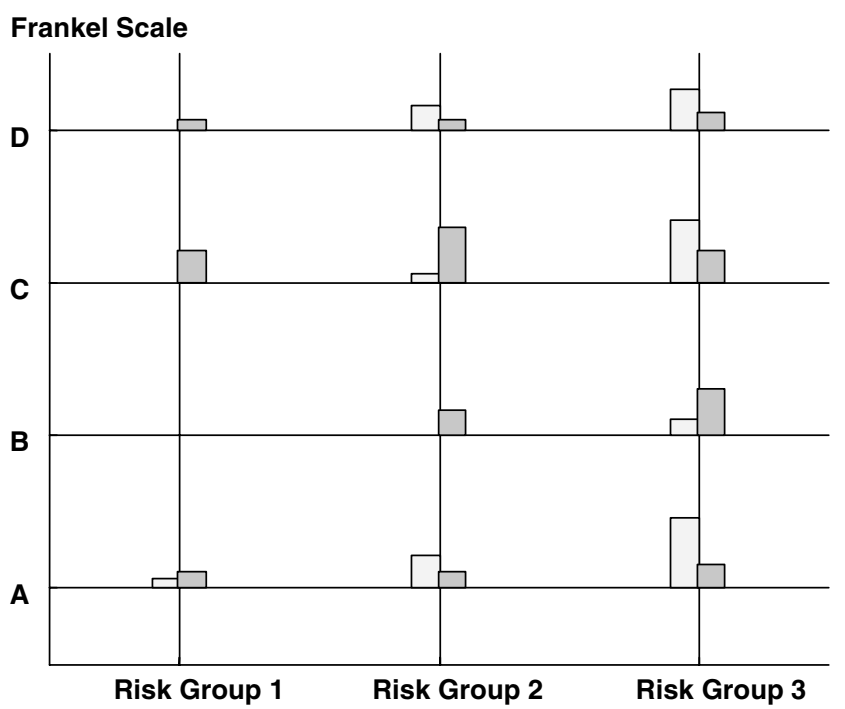

Figure 5 Frequency of survival time (line pattern: $\leqslant 10$ months; dot pattern: $>10$ months) dependent on tumour risk groups I, II and III and Frankel classification (A, B, C, D)

consecutive patients confirm these findings. Major improvements were achieved during the inpatient rehabilitation, especially in aspects of self-care and mobility. This is reflected by an increase in the FIM score from 64 to 82 . Considering the length of stay, an efficiency of rehabilitation of 0.33 per day was computed. As expected, this is lower than the estimated efficiency for trauma patients $(0.86-1.07)$, but within the range reported for nontraumatic complete paraplegic patients $(0.42){ }^{20}$

It was our intention to conclude a basic rehabilitation programme within 4-6 weeks. However, the length of stay proved to be subject to several variables. The pre-existence of pressure sores was a major factor: these patients $(n=8)$ required inpatient care for an average of 123 days. Therefore, it should be a minimum requirement for primary treatment facilities to provide adequate nursing care, and hence to prevent such pressure sores.

The expected remaining life span is one of the key factors for the investment of rehabilitative efforts and resources. The public administration registration of all citizens allowed a complete follow-up for all patients. The median survival of our patients was 11 months after the onset of neurologic symptoms. We expected tumour type and level of lesion to be the key factors determining longer survival. After completion of statistical analysis using two different approaches, the FIM score at the time of admission proved to be the most reliable predictive variable. The FIM score reflects the general clinical situation of a patient. Therefore, the indication for rehabilitative efforts should be based on the patient's clinical status at the time of presentation. Tumour type and level of lesion should also be considered, but were less potent as indicators of survival in our series. The observed longer survival of female patients may be explained by the distribution of tumour types (less aggressive for females).

Recently, Tomita $\mathrm{et}_{\mathrm{al}} \mathrm{p}^{7}$ presented a prognostic scoring system for the indication of primary surgical treatment: tumour aggressiveness and evidence as well as localisation of metastases were identified as prognostic factors. In their study, only 34 of the 61 patients had neurologic deficits, and postoperative improvement was noted in 25 patients. The follow-up or the functional status of the remaining patients with SCI is not addressed further. ${ }^{7}$

We were not able to obtain retrospectively the definitive oncological status of all our patients (evidence of visceral, pulmonary or other bone metastases) because there was no standard protocol for the staging of the tumour and the search for metastases. We therefore cannot comment on the impact of metastases towards survival time. However, we expect that a combination of both functional and oncological parameters will give the best survival prognosis. Patients with metastatic disease of the spine are subject to extensive surgery, ${ }^{3-8}$ sophisticated chemotherapy ${ }^{11,12}$ or radiation protocols. ${ }^{9,10}$ Consequently, SCI - once it is evident that neurological symptoms will not regress should not be answered with rehabilitative nihilism. On the contrary, according to our data and the data already published concerning the rehabilitation efficiency, ${ }^{26}$ patients who are in acceptable clinical status will benefit from an intensive inpatient rehabilitation programme of a dedicated SCI unit.

\section{References}

1 Black P. Spinal metastasis: current status and recommended guidelines for management. Neurosurgery 1979; 5: 726-746.

2 Bach $\mathrm{F}$ et al. Metastatic spinal cord compression. Occurrence, symptoms, clinical presentations and prognosis in 398 patients with spinal cord compression. Acta Neurochir (Wien) 1990; 107: 37-43. 
3 Harrington KD. Anterior decompression and stabilization of the spine as a treatment for vertebral collapse and spinal cord compression from metastatic malignancy. Clin Orthop 1988; 233: 177-197.

4 Kluger P, Korge A, Scharf HP. Strategy for the treatment of patients with spinal neoplasms. Spinal Cord 1997; 35: 429-436.

5 Rompe JD, Eysel P, Hopf C, Heine J. Decompression/ stabilization of the metastatic spine. Cotrel-DuboussetInstrumentation in 50 patients. Acta Orthop Scand 1993; 64: $3-8$.

6 Sundaresan $\mathrm{N}$ et al. Indications and results of combined anterior-posterior approaches for spine tumor surgery. $J$ Neurosurg 1996; 85: 438-446.

7 Tomita K et al. Surgical strategy for spinal metastases. Spine 2001; 26: 298-306.

8 Wise $\mathbf{J} \mathbf{J}$ et al. Complication, survival rates, and risk factors of surgery for metastatic disease of the spine. Spine 1999; 24: 1943-1951.

9 Helweg-Larsen S, Johnsen A, Boesen J, Sorensen PS. Radiologic features compared to clinical findings in a prospective study of 153 patients with metastatic spinal cord compression treated by radiotherapy. Acta Neurochir (Wien) 1997; 139: 105-111.

10 Helweg-Larsen S. Clinical outcome in metastatic spinal cord compression. A prospective study of 153 patients. Acta Neurol Scand 1996; 94: 269-275.

11 Diener KM. Bisphosphonates for controlling pain from metastatic bone disease. Am J Health Syst Pharm 1996; 53: 1917-1927.

12 Eeles RA, O'Brien P, Horwich A, Brada M. NonHodgkin's lymphoma presenting with extradural spinal cord compression: functional outcome and survival. $\mathrm{Br} \mathrm{J}$ Cancer 1991; 63: 126-129.

13 Ditunno Jr JF, Young W, Donovan WH, Creasey G. The international standards booklet for neurological and functional classification of spinal cord injury. American Spinal Injury Association. Paraplegia 1994; 32: 70-80.
14 Zadeh LA. Fuzzy sets. Inform Control 1965; 8: 338-353.

15 Jäkel J, Gröll L, Mikut R. Automatic generation and evaluation of interpretable rule bases for fuzzy systems. In: Mohammadian M (ed). New Frontiers in Compuational Intelligence and its Applications. IOS Press: Amsterdam 2000, pp 1-10.

16 Mikut R, Jäkel J, Malberg H, Bretthauer G. Datenbasierter Entwurf von Fuzzy-Systemen für medizinische Diagnoseaufgaben. Automatisierungstechnik 2000; 48: 317-326.

17 Ewerbeck V, Friedl W. Chirurgische Therapie von Skelettmetastasen. Springer: Heidelberg, 1992.

18 Noltenius H. Tumor Handbuch: Pathologie und Klinik der menschlichen Tumoren. Urban and Schwarzenberg: München, 1987.

19 Tatsui $\mathrm{H}$ et al. Survival rates of patients with metastatic spinal cancer after scintigraphic detection of abnormal radioactive accumulation. Spine 1996; 21: 2143-2148.

20 McKinley WO, Sell RT, Hardman JT. Nontraumatic spinal cord injury: incidence, epidemiology, and functional outcome. Arch Phys Med Rehabil 1999; 80: 619-623.

21 DeVivo MJ et al. The influence of age at time of spinal cord injury on rehabilitation outcome. Arch Neurol 1990; 47: 687-691.

22 Devivo MJ, Richards JS, Stover SL, Go BK. Spinal cord injury. Rehabilitation adds life to years. West J Med 1991; 154: $602-606$.

23 DeVivo MJ et al. Trends in spinal cord injury demographics and treatment outcomes between 1973 and 1986. Arch Phys Med Rehabil 1992; 73: 424-430.

24 Gerner HJ. Die Querschnittlähmung. Blackwell: Berlin, 1992.

25 Bhalla SK. Metastatic disease of the spine. Clin Orthop 1970; 73: 52-60.

26 McKinley WO, Conti-Wyneken AR, Vokac CW, Cifu DX. Rehabilitative functional outcome of patients with neoplastic spinal cord compressions. Arch Phys Med Rehabil 1996; 77: 892-895. 